Supporting Information for

\title{
Yielding Criteria of Double Network Hydrogels
}

Takahiro Matsuda, Tasuku Nakajima, Yuki Fukuda, Wei Hong, Takamasa Sakai, Takayuki Kurokawa, Ung-il Chung, and Jian Ping Gong

\section{Calculation of contour length of the tetra-PEG network strand:}

Molecular weight of the tetra-PEG network strand is $5,000(\mathrm{~g} / \mathrm{mol})$. If we neglect an amine bond at the center of a chain for simplicity, this molecular weight corresponds to 113.6 monomer units. Bond angles and lengths in a monomer unit were estimated from the molecular modeling results of triethylene glycol. The minimum energy configuration of triethylene glycol was performed using an MM2 force field with the software ChemBioDraw 12.0 (Cambridgesoft, Cambridge, MA). As a result, the following values were calculated.

Average C-O-C bond angle: $110.6^{\circ}$

Average O-C-C bond angle: $105.8^{\circ}$

Average $\mathrm{C}-\mathrm{O}$ bond length: $1.458 \AA$

Average C-C bond length: $1.534 \AA$

With an assumption of constant bond angle and length and all-trans conformation of PEG, contour length of the TPEG network strand can be calculated as $41 \mathrm{~nm}$. 
Molecular weight distribution of the tetra-PEG macromer:

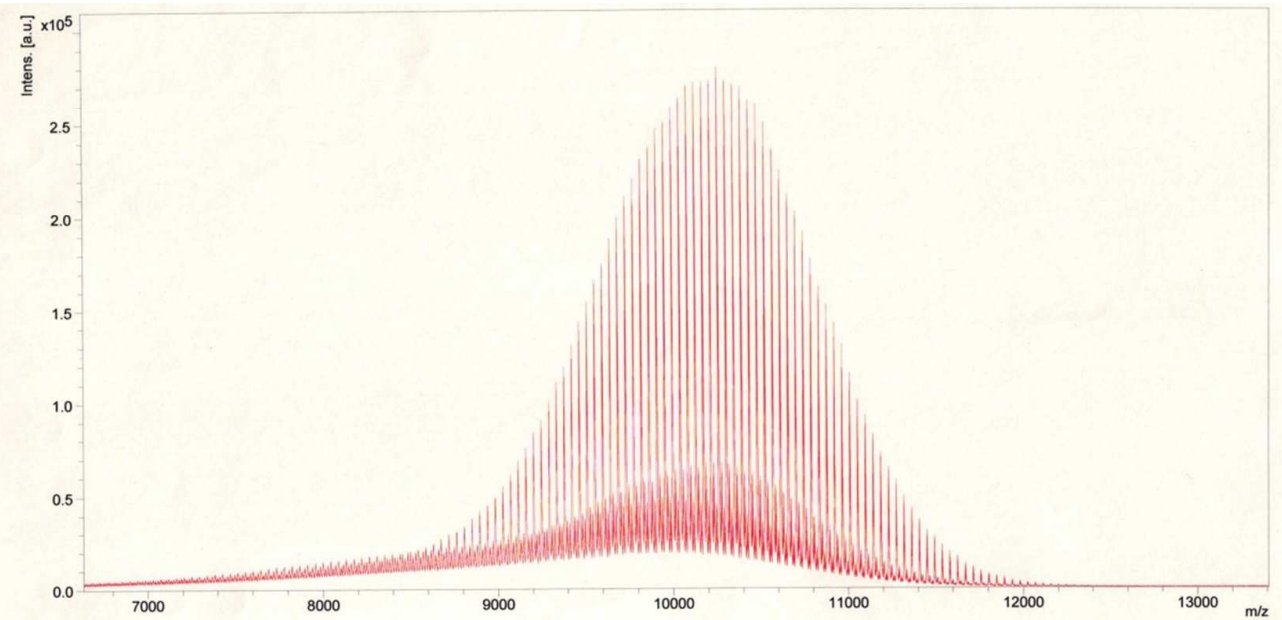

Figure S1: A MALDI-TOF spectrum of the tetra-amine-terminated PEG $(M w: 10 \mathrm{~kg} / \mathrm{mol}, M w / M n=$ 1.05). 